\title{
EXISTENCE OF SOLUTION FOR A SINGULAR ELLIPTIC EQUATION WITH CRITICAL SOBOLEV-HARDY EXPONENTS
}

\author{
JUAN LI
}

Received 9 June 2005 and in revised form 21 September 2005

Via the variational methods, we prove the existence of a nontrivial solution to a singular semilinear elliptic equation with critical Sobolev-Hardy exponent under certain conditions.

\section{Introduction}

In this paper, we consider the following elliptic problem:

$$
-\Delta u-\mu \frac{u}{|x|^{2}}=\frac{|u|^{2 *(s)-2}}{|x|^{s}} u+a(x)|u|^{r-2} u+\lambda u, \quad x \in \mathbb{R}^{N}
$$

where $N \geq 3,0 \leq \mu<\bar{\mu} \doteq((N-2) / 2)^{2}, 0 \leq s<2, \lambda \geq 0$, and $2^{*}(s) \doteq 2(N-s) /(N-$ $2)$ is the critical Sobolev-Hardy exponent; note that $2^{*}(0)=2^{*} \doteq 2 N /(N-2)$ is the critical Sobolev exponent. The space $H \doteq H\left(\mathbb{R}^{N}\right)$ is the completion of $C_{0}^{\infty}\left(\mathbb{R}^{N}\right)$ in the norm

$$
\|u\| \doteq\left(\int_{\mathbb{R}^{N}}\left(|\nabla u|^{2}-\mu \frac{u^{2}}{|x|^{2}}\right) d x\right)^{1 / 2}
$$

By the Hardy inequality $[8,9]$, this norm is equivalent to the usual norm $\left(\int_{\mathbb{R}^{N}}|\nabla u|^{2} d x\right)^{1 / 2}$. The scalar product in $H$ is

$$
(u, v) \doteq \int_{\mathbb{R}^{N}}\left(\nabla u \nabla v-\mu \frac{u v}{|x|^{2}}\right) d x \quad \forall u, v \in H
$$

We define $H_{r} \subset H$ with

$$
H_{r} \doteq\{u \in H, u(x)=u(|x|)\}
$$




\section{A solution to a singular critical elliptic equation}

The hypothesis for $a(x)$ is as follows:

(A) $a(x)$ is nonnegative and locally bounded in $\mathbb{R}^{N} \backslash\{0\}, a(x)=O\left(|x|^{-s}\right)$ in the bounded neighborhood $G$ of the origin, $a(x)=O\left(|x|^{-t}\right)$ as $|x| \rightarrow \infty, 0 \leq s<t<2,2^{*}(t)<$ $r<2 *(s)$, where $2^{*}(t) \doteq 2(N-t) /(N-2)$ for $0 \leq t<2$.

The singular elliptic problems have received some attention in recent years. For example, Janneli [10] and Ferrero and Gazzolo [7] studied the semilinear elliptic equation

$$
\begin{gathered}
-\Delta u-\mu \frac{u}{|x|^{2}}=|u|^{2^{*}-2} u+\lambda u, \quad x \in \Omega, \\
u(x)=0, \quad x \in \partial \Omega,
\end{gathered}
$$

where $\Omega \subset \mathbb{R}^{N}(N \geq 3)$ is a smooth bounded domain containing the origin 0 . They proved that (1.5) has a nontrivial solution under certain conditions for $\lambda$ and $\mu$. Moreover, Cao in $[4,5]$ and Chen in [6] also studied the semilinear elliptic equation (1.5). They show that (1.5) has nontrivial solutions and a sign-changing solution under some conditions for $\mu, \lambda$. Ghoussoub and Yuan in [9] considered the quasilinear problem

$$
\begin{gathered}
-\Delta_{p} u=\mu \frac{|u|^{q-2} u}{|x|^{s}}+\lambda|u|^{r-2} u, \quad x \in \Omega, \\
u(x)=0, \quad x \in \partial \Omega .
\end{gathered}
$$

They get that (1.6) has a positive solution and a sign-changing solution under some conditions for $\lambda, \mu, r, q$.

In the case when $\Omega$ is an unbounded domain in $\mathbb{R}^{N}$, the corresponding problem becomes more complicated since the Sobolev embedding $W^{1, p}(\Omega) \hookrightarrow L^{q}(\Omega)(p \geq 2)$ is not compact for all $q \in\left[p, p^{*}\right]$. However, by the Strauss lemma (see [13]), the embedding $H_{r}\left(\mathbb{R}^{N}\right) \hookrightarrow L^{q}\left(\mathbb{R}^{N}\right)$ is compact for all $q \in\left[2,2^{*}\right)$. Therefore, we can discuss the nontrivial solutions of (1.1) in $H_{r}$ by variational methods. But there are also some difficulties for (1.1), because the embedding $H_{r} \hookrightarrow L^{2^{*}(s)}\left(\mathbb{R}^{N},|x|^{-s}\right)$ is still not compact. In [11], as $\lambda=0$, the existence of a nontrivial solution is given for (1.1) with $s=0$, so it will be meaningful to study the existence of nontrivial solutions for $(1.1)$ as $s \in[0,2)$ and $\lambda \neq 0$. In this paper, we obtain the following existence results.

Theorem 1.1. Suppose (A) and $0 \leq s<2,0 \leq \mu<\bar{\mu}, \lambda \geq 0$. Assume that one of the following conditions holds:

(i) $\lambda=0$ and

$$
\max \left\{\frac{N-s}{\sqrt{\bar{\mu}}+\sqrt{\bar{\mu}-\mu}}, \frac{N-s-2 \sqrt{\bar{\mu}-\mu}}{\sqrt{\bar{\mu}}}, 2^{*}(t)\right\}<r<2^{*}(s),
$$

(ii) $0<\lambda<\lambda_{1}(\mu)$ and $0 \leq \mu \leq \bar{\mu}-1$, where $\lambda_{1}(\mu) \doteq \inf _{u \in H \backslash\{0\}}\left(\|u\|^{2} / \int_{\mathbb{R}^{N}} u^{2} d x\right)$. Then problem (1.1) has at least a nontrivial solution in $H_{r}$.

Throughout this paper, we will use the letter $C$ to denote the natural various constants independent of $u$, and $\int \cdot d x$ instead of $\int_{\mathbb{R}^{N}} \cdot d x$. 


\section{Proof of the main result}

We first give some definitions and lemmas.

Definition 2.1. Let $\left\{u_{m}\right\}$ be a sequence in $H_{r}$, if there exists a constant $c \in \mathbb{R}^{1}$ such that

$$
J\left(u_{m}\right) \longrightarrow c, \quad J^{\prime}\left(u_{m}\right) \longrightarrow 0 \quad \text { in } H_{r}^{-1}
$$

as $m \rightarrow \infty$, then $\left\{u_{m}\right\}$ is called a (PS) sequence in $H_{r}$.

Lemma 2.2 (Hardy inequality $[8,9]$ ). Assume that $1<p<N$ and $u \in W^{1, p}\left(\mathbb{R}^{N}\right)$. Then

$$
\int \frac{|u|^{p}}{|x|^{p}} d x \leq\left(\frac{p}{N-p}\right)^{p} \int|\nabla u|^{p} d x
$$

Lemma 2.3 (Sobolev-Hardy inequality [9]). Assume that $1<p<N$ and that $p^{*}(s) \doteq$ $((N-s) /(N-p)) p, 0 \leq s \leq p$. Then there exists a constant $C>0$ such that for any $u \in$ $W^{1, p}\left(\mathbb{R}^{N}\right)$,

$$
\left(\int \frac{\left.|u|\right|^{*}(s)}{|x|^{s}} d x\right)^{p / p^{*}(s)} \leq C \int|\nabla u|^{p} d x
$$

Lemma 2.4 [11]. Assume that hypothesis (A) holds. Then the embedding $H \hookrightarrow L^{r}\left(\mathbb{R}^{N}, a(x)\right)$ is compact.

Consider the energy functional

$$
J(u)=\frac{1}{2}\|u\|-\frac{1}{2 *(s)} \int \frac{|u|^{2^{*}(s)}}{|x|^{s}} d x-\frac{1}{r} \int a(x)|u|^{r} d x-\frac{\lambda}{2} \int|u|^{2} d x,
$$

by Lemma 2.4, $J(u)$ is well defined and $J \in C^{1}(H, \mathbb{R})$; the critical points of the functional $J$ correspond to weak solutions of problem (1.1).

For $0 \leq \mu<\bar{\mu}$, define the best Sobolev-Hardy constant:

$$
A_{s} \doteq A_{s}(\mu)=\inf _{u \in H\{0\}} \frac{\int\left(|\nabla u|^{2}-\mu u^{2} /|x|^{2}\right) d x}{\left(\int|u|^{2 *(s)} /|x|^{s} d x\right)^{2 / 2^{*}(s)}}
$$

In $[12]$, the author found that $A_{s}$ is attained by the functions

$$
y_{\varepsilon}(x)=\frac{(2 \varepsilon(\bar{\mu}-\mu)(N-s) / \sqrt{\bar{\mu}})^{\sqrt{\bar{\mu}} /(2-s)}}{|x|^{\sqrt{\bar{\mu}}-\sqrt{\bar{\mu}-\mu}}\left(\varepsilon+|x|^{(2-s) \sqrt{\bar{\mu}-\mu} / \sqrt{\bar{\mu}}}\right)^{(N-2) /(2-s)}}
$$

for all $\varepsilon>0$. Moreover, the functions $y_{\varepsilon}(x)$ solve the equation

$$
-\Delta u-\mu \frac{u}{|x|^{2}}=\frac{|u|^{2 *(s)-2}}{|x|^{s}} u \quad \text { in } \mathbb{R}^{N} \backslash\{0\}
$$


3216 A solution to a singular critical elliptic equation

and satisfy

$$
\int\left(\left|\nabla y_{\varepsilon}\right|^{2}-\mu \frac{\left|y_{\varepsilon}\right|^{2}}{|x|^{2}}\right) d x=\int \frac{\left|y_{\varepsilon}\right|^{2^{*}(s)}}{|x|^{s}} d x=A_{s}^{(N-s) /(2-s)} .
$$

In the following, we first give some estimates for the extremal functions.

Let

$$
C_{\varepsilon}=\left(\frac{2 \varepsilon(\bar{\mu}-\mu)(N-s)}{\sqrt{\bar{\mu}}}\right)^{\sqrt{\bar{\mu} /(2-s)}}, \quad U_{\varepsilon}(x)=\frac{y_{\varepsilon}(x)}{C_{\varepsilon}}
$$

$B_{2 l}=\left\{x \in \mathbb{R}^{N},|x|<2 l\right\} \subset G$ with $l>0$ and $G$ is the domain in hypothesis (A), let $0 \leq \phi \leq$ 1 be a cutting-off function in $C_{0}^{\infty}\left(\mathbb{R}^{N}\right) \cap H_{r}$, such that $\phi(x)=1$ in $B_{l}$ and $\phi(x)=0$ in $\mathbb{R}^{N} \backslash$ $B_{2 l}$. Set $u_{\varepsilon}(x)=\phi(x) y_{\varepsilon}(x)$ and $v_{\varepsilon}=u_{\varepsilon}(x) /\left(\int\left|u_{\varepsilon}\right|^{2^{*}(s)} /|x|^{s}\right)^{1 / 2^{*}(s)}$, so that $\int\left(\left|v_{\varepsilon}\right|^{2^{*}(s)} /|x|^{s}\right)=$ 1. In [12], the author proved that the following estimates are true:

$$
\int\left|v_{\varepsilon}\right|^{q} d x= \begin{cases}O\left(\varepsilon^{\sqrt{\mu} q /(2-s)}\right), & 1 \leq q<\frac{N}{\sqrt{\bar{\mu}}+\sqrt{\bar{\mu}-\mu}}, \\ O\left(\varepsilon^{\sqrt{\mu} q /(2-s)}|\ln \varepsilon|\right), & q=\frac{N}{\sqrt{\bar{\mu}}+\sqrt{\bar{\mu}-\mu}}, \\ O\left(\varepsilon^{\sqrt{\mu}(N-q \sqrt{\bar{\mu}}) /((2-s) \sqrt{\bar{\mu}-\mu})}\right), & \frac{N}{\sqrt{\bar{\mu}}+\sqrt{\bar{\mu}-\mu}}<q<2^{*} .\end{cases}
$$

Moreover, we also need the following results.

Lemma 2.5. Suppose that $\gamma=\sqrt{\bar{\mu}}+\sqrt{\bar{\mu}-\mu}, \gamma=\sqrt{\bar{\mu}}-\sqrt{\bar{\mu}-\mu}, 0 \leq \mu<\bar{\mu}$, and $0 \leq s<2$, then, $v_{\varepsilon}(x)$ satisfies the following estimates:

$$
\int \frac{\left|v_{\varepsilon}\right|^{q}}{|x|^{s}} d x \geq \begin{cases}c_{1} \varepsilon^{\sqrt{\mu} q /(2-s)}, & 1 \leq q<\frac{N-s}{\gamma}, \\ c_{2} \varepsilon^{\sqrt{\mu} q /(2-s)}|\ln \varepsilon|, & q=\frac{N-s}{\gamma}, \\ c_{3} \varepsilon^{(\sqrt{\bar{\mu}}(N-s)-\bar{\mu} q) /(2-s) \sqrt{\bar{\mu}-\mu},} & \frac{N-s}{\gamma}<q<2^{*}(s),\end{cases}
$$

where $c_{i}(i=1,2,3)$ are positive constants. 
Proof. Let $\omega_{N}$ denote the surface area of the $(N-1)$ sphere $S^{N-1}$ in $\mathbb{R}^{N}$. For $1 \leq q<2^{*}(s)$, we have

$$
\begin{aligned}
\int \frac{\left|v_{\varepsilon}\right|^{q}}{|x|^{s}} d x= & \int \frac{\left|u_{\varepsilon}(x)\right|^{q}}{|x|^{s}} d x \cdot\left(\int \frac{\left|u_{\varepsilon}\right|^{2^{*}(s)}}{|x|^{s}} d x\right)^{-q / 2^{*}(s)}=B \int \frac{\left|\phi(x) C_{\varepsilon} U_{\varepsilon}\right|^{q}}{|x|^{s}} d x \\
= & B C_{\varepsilon}^{q}\left(O(1)+\omega_{N} \int_{0}^{l}\left(\varepsilon+r^{(2-s) \sqrt{\mu}-\mu / \sqrt{\bar{\mu}}}\right)^{-q(N-2) /(2-s)} r^{N-s-1-q \hat{\gamma}} d r\right) \\
= & B C_{\varepsilon}^{q}\left(O(1)+\omega_{N} \varepsilon^{-q((N-2) /(2-s))+(\sqrt{\bar{\mu}}(N-s-\gamma q) /(2-s) \sqrt{\bar{\mu}-\mu})}\right. \\
& \left.\quad \times \int_{0}^{l \varepsilon \sqrt{\bar{\mu} /((s-2) \sqrt{\mu}-\mu)}}\left(1+r^{(2-s) \sqrt{\bar{\mu}-\mu} / \sqrt{\bar{\mu}}}\right)^{-q(N-2) /(2-s)} r^{N-s-1-q \hat{\gamma}} d r\right),
\end{aligned}
$$

where $B=\left(\int\left|u_{\varepsilon}\right|^{2^{*}(s)} /|x|^{s} d x\right)^{-q / 2^{*}(s)}$.

If $-2 q \sqrt{\bar{\mu}-\mu}+N-s-\hat{\gamma} q=0$, that is, $q=(N-s) / \gamma$,

$$
\int \frac{\left|v_{\varepsilon}\right|^{q}}{|x|^{s}} d x=B C_{\varepsilon}^{q}\left(O(1)+\omega_{N} \int_{1}^{\left.l \varepsilon^{\sqrt{\mu} /(s-2) \sqrt{\mu}-\mu}\right)} \frac{1}{r} d r\right) \geq B c_{1} \varepsilon^{\sqrt{\mu} q /(2-s)}|\ln \varepsilon|,
$$

where $\dot{c}_{1}>0$ is a constant.

If $-2 q \sqrt{\bar{\mu}-\mu}+N-s-\dot{\gamma} q<0$, that is, $q>(N-s) / \gamma$,

$$
\begin{aligned}
\int \frac{\left|v_{\varepsilon}\right|^{q}}{|x|^{s}} d x & =B C_{\varepsilon}^{q}\left(O(1)+O\left(\varepsilon^{-q((N-2) /(2-s))+(\sqrt{\bar{\mu}}(N-s-\hat{\gamma} q) /(2-s) \sqrt{\bar{\mu}-\mu})}\right)\right) \\
& \geq B c_{2} \varepsilon^{(\sqrt{\mu}(N-s)-\bar{\mu} q) /(2-s) \sqrt{\bar{\mu}-\mu}}
\end{aligned}
$$

where $c_{2}^{\prime}>0$ is a constant.

If $-2 q \sqrt{\bar{\mu}-\mu}+N-s-\gamma q>0$, that is, $q<(N-s) / \gamma$,

$$
\begin{aligned}
\int \frac{\left|v_{\varepsilon}\right|^{q}}{|x|^{s}} d x & =B C_{\varepsilon}^{q}\left(O(1)+\omega_{N} \int_{0}^{l}\left(\varepsilon+r^{(2-s) \sqrt{\bar{\mu}-\mu} / \sqrt{\bar{\mu}}}\right)^{-q(N-2) /(2-s)} r^{N-s-1-q \hat{\gamma}} d x\right) \\
& =B C_{\varepsilon}^{q} \cdot O(1) \geq B c_{3} \varepsilon^{\sqrt{\bar{\mu}} q /(2-s)},
\end{aligned}
$$

where $\dot{c}_{3}>0$ is a constant.

By

$$
\begin{aligned}
B=\left(\int \frac{\left|u_{\varepsilon}\right|^{2^{*}(s)}}{|x|^{s}} d x\right)^{-q / 2^{*}(s)} & =\left(\int \frac{\left|\phi(x) y_{\varepsilon}\right|^{2^{*}(s)}}{|x|^{s}} d x\right)^{-q / 2^{*}(s)} \\
& \geq\left(\int \frac{\left|y_{\varepsilon}\right|^{2^{*}(s)}}{|x|^{s}} d x\right)^{-q / 2^{*}(s)}=A_{s}^{(2-N) q / 2(2-s)},
\end{aligned}
$$

we have finished the proof of Lemma 2.5. 
3218 A solution to a singular critical elliptic equation

Lemma 2.6. Suppose (A) and $0 \leq s<2,0 \leq \mu<\bar{\mu}, \lambda \geq 0$. Assume that one of the following conditions holds:

(i) $\lambda=0$ and

$$
\max \left\{\frac{N-s}{\sqrt{\bar{\mu}}+\sqrt{\bar{\mu}-\mu}}, \frac{N-s-2 \sqrt{\bar{\mu}-\mu}}{\sqrt{\bar{\mu}}}, 2^{*}(t)\right\}<r<2^{*}(s),
$$

(ii) $0<\lambda<\lambda_{1}(\mu)$ and $0 \leq \mu \leq \bar{\mu}-1$.

Then, there exists $u_{0} \in H_{r}, u_{0} \neq 0$, such that the following inequality holds:

$$
0<\sup _{t \geq 0} J\left(t u_{0}\right)<\frac{2-s}{2(N-s)} A_{s}^{(N-s) /(2-s)}
$$

Proof. For $t \geq 0$, we consider the functions

$$
\begin{gathered}
g(t) \doteq J\left(t v_{\varepsilon}\right)=\frac{t^{2}}{2}\left\|v_{\varepsilon}\right\|^{2}-\frac{t^{2^{*}(s)}}{2^{*}(s)}-\frac{t^{r}}{r} \int a(x)\left|v_{\varepsilon}\right|^{r} d x-\frac{\lambda t^{2}}{2} \int\left|v_{\varepsilon}\right|^{2} d x, \\
\bar{g}(t)=\frac{t^{2}}{2}\left\|v_{\varepsilon}\right\|^{2}-\frac{t^{2^{*}(s)}}{2^{*}(s)} .
\end{gathered}
$$

Note that $\lim _{t \rightarrow \infty} g(t)=-\infty, g(0)=0$, and $g(t)>0$ as $t \rightarrow 0^{+}$, therefore, $\sup _{t \geq 0} g(t)>0$ must be attained by some $0<t_{\varepsilon}<+\infty$ and $g^{\prime}\left(t_{\varepsilon}\right)=0$. So we have

$$
g^{\prime}\left(t_{\varepsilon}\right)=t_{\varepsilon}|| v_{\varepsilon} \|^{2}-t_{\varepsilon}^{2^{*}(s)-1}-t_{\varepsilon}^{r-1} \int a(x)\left|v_{\varepsilon}\right|^{r} d x-\lambda t_{\varepsilon} \int\left|v_{\varepsilon}\right|^{2} d x=0
$$

Then

$$
\left\|v_{\varepsilon}\right\|^{2}=t_{\varepsilon}^{2^{*}(s)-2}+t_{\varepsilon}^{r-2} \int a(x)\left|v_{\varepsilon}\right|^{r} d x+\lambda \int\left|v_{\varepsilon}\right|^{2} d x \geq t_{\varepsilon}^{2^{*}(s)-2}, \quad t_{\varepsilon} \leq\left\|v_{\varepsilon}\right\|^{2 /\left(2^{*}(s)-2\right)} .
$$

Moreover, by hypothesis (A), we have

$$
\left\|v_{\varepsilon}\right\|^{2} \leq t_{\varepsilon}^{2^{*}(s)-2}+C|| v_{\varepsilon} \|^{2(r-2) /\left(2^{*}(s)-2\right)} \int_{B_{2 l}} \frac{\left|v_{\varepsilon}\right|^{r}}{|x|^{s}}+\lambda \int\left|v_{\varepsilon}\right|^{2} d x .
$$

From (2.23) and (2.10)-(2.12), as $\varepsilon$ small enough, we get

$$
t_{\varepsilon}^{2^{*}(s)-2} \geq \frac{A_{s}}{2}
$$

By the simple computation, we know that the function $\bar{g}(t)$ attains its maximum at $t_{0}=\left\|v_{\varepsilon}\right\|^{2 /\left(2^{*}(s)-2\right)}$ and is increasing in the interval $\left[0, t_{0}\right]$. So, by $(2.10),(2.22)$, and (2.24), 
we have

$$
\begin{aligned}
g\left(t_{\varepsilon}\right) & \leq \bar{g}\left(t_{0}\right)-\frac{1}{r}\left(\frac{A_{s}}{2}\right)^{r /\left(2^{*}(s)-2\right)} \int \frac{\left|v_{\varepsilon}\right|^{r}}{|x|^{s}} d x-\frac{\lambda}{2}\left(\frac{A_{s}}{2}\right)^{2 /\left(2^{*}(s)-2\right)} \int\left|v_{\varepsilon}\right|^{2} d x \\
& \leq \frac{2-s}{2(N-s)} \|\left. v_{\varepsilon}\right|^{2(N-s) /(2-s)}-C \int \frac{\left|v_{\varepsilon}\right|^{r}}{|x|^{s}}-C \int\left|v_{\varepsilon}\right|^{2} d x \\
& =\frac{2-s}{2(N-s)} A_{s}^{(N-s) /(2-s)}+O\left(\varepsilon^{(N-2) /(2-s)}\right)-C \int \frac{\left|v_{\varepsilon}\right|^{r}}{|x|^{s}}-C \int\left|v_{\varepsilon}\right|^{2} d x .
\end{aligned}
$$

In case (i), since

$$
r>\max \left\{\frac{N-s}{\gamma}, \frac{N-s-2 \sqrt{\bar{\mu}-\mu}}{\sqrt{\bar{\mu}}}, 2^{*}(t)\right\},
$$

by (2.12), we have

$$
\begin{gathered}
\int \frac{\left|v_{\varepsilon}\right|^{r}}{|x|^{s}} \geq c_{3} \varepsilon \sqrt{\bar{\mu}}(N-s-\sqrt{\bar{\mu}} r) /(2-s) \sqrt{\bar{\mu}-\mu}, \\
\frac{\sqrt{\bar{\mu}}(N-s-\sqrt{\bar{\mu}} r)}{(2-s) \sqrt{\bar{\mu}-\mu}}<\frac{N-2}{2-s} .
\end{gathered}
$$

Let $u_{0}=v_{\varepsilon}$, choosing $\varepsilon$ small enough, from (2.25), we can deduce that

$$
\sup _{t \geq 0} J\left(t u_{0}\right)=g\left(t_{\varepsilon}\right)<\frac{2-s}{2(N-s)} A_{s}^{(N-s) /(2-s)} .
$$

In case (ii), $0<\lambda<\lambda_{1}(\mu)$. By (2.11), as $\mu=\bar{\mu}-1$,

$$
\int\left|v_{\varepsilon}\right|^{2}=O\left(\varepsilon^{(N-2) /(2-s)}|\ln \varepsilon|\right),
$$

as $0 \leq \mu<\bar{\mu}-1$,

$$
\int\left|v_{\varepsilon}\right|^{2}=O\left(\varepsilon^{(N-2) /((2-s) \sqrt{\bar{\mu}-\mu})}\right) .
$$

Choosing $\varepsilon$ small enough, we also get (2.28). The proof of Lemma 2.6 is completed.

Lemma 2.7. Suppose that $c \in\left(0,(2-s) /(2(N-s)) A_{s}^{(N-s) /(2-s)}\right)$. Then $J(u)$ satisfies $(\mathrm{PS})_{c}$ condition.

Proof. Let $\left\{u_{m}\right\} \in H_{r}$ be a $(\mathrm{PS})_{c}$ sequence. Then we have

$$
\begin{aligned}
& J\left(u_{m}\right)=\frac{1}{2}\left\|u_{m}\right\|^{2}-\frac{1}{2^{*}(s)} \int \frac{\left|u_{m}\right|^{2^{*}(s)}}{|x|^{s}} d x-\frac{1}{r} \int a(x)\left|u_{m}\right|^{r} d x-\frac{\lambda}{2} \int\left|u_{m}\right|^{2} d x=c+o(1), \\
& \left\langle J^{\prime}\left(u_{m}\right), u_{m}\right\rangle=\left\|u_{m}\right\|^{2}-\int \frac{\left|u_{m}\right|^{2^{*}(s)}}{|x|^{s}} d x-\int a(x)\left|u_{m}\right|^{r} d x-\lambda \int\left|u_{m}\right|^{2} d x=o(1)\left\|u_{m}\right\| .
\end{aligned}
$$


3220 A solution to a singular critical elliptic equation

Let $(2.31) \times 2-(2.32)$, we have

$$
2 c+o(1)+o(1)\left\|u_{m}\right\| \geq\left(1-\frac{2}{2^{*}(s)}\right) \int \frac{\left|u_{m}\right|^{2^{*}(s)}}{|x|^{s}} d x+\left(1-\frac{2}{r}\right) \int a(x)\left|u_{m}\right|^{r} d x
$$

From

$$
\left\|u_{m}\right\|^{2}=2 J\left(u_{m}\right)+\frac{2}{2^{*}(s)} \int \frac{\left|u_{m}\right|^{2^{*}(s)}}{|x|^{s}} d x+\frac{2}{r} \int a(x)\left|u_{m}\right|^{r} d x+\lambda \int\left|u_{m}\right|^{2} d x,
$$

we get

$$
\begin{aligned}
\left(1-\frac{\lambda}{\lambda_{1}(\mu)}\right)\left\|u_{m}\right\|^{2} & \leq 2 J\left(u_{m}\right)+\frac{2}{2^{*}(s)} \int \frac{\left|u_{m}\right|^{2^{*}(s)}}{|x|^{s}} d x+\frac{2}{r} \int a(x)\left|u_{m}\right|^{r} d x \\
& \leq o(1)+o(1)\left\|u_{m}\right\|+C .
\end{aligned}
$$

So, we conclude that $\left\{u_{m}\right\}$ is bounded in $H_{r}$. Passing to a subsequence (still denoted by $\left.\left\{u_{m}\right\}\right)$, as $m \rightarrow \infty$, we get that

$$
\begin{aligned}
& u_{m} \rightarrow u \text { weakly in } H_{r}, \\
& u_{m} \longrightarrow u \text { strongly in } L^{q}\left(\mathbb{R}^{N}\right), \quad q \in\left[2,2^{*}\right), \\
& u_{m} \longrightarrow u \text { a.e. in } \mathbb{R}^{N}, \\
& u_{m} \longrightarrow u \text { strongly in } L^{r}\left(\mathbb{R}^{N}, a(x)\right) .
\end{aligned}
$$

It follows from the Sobolev-Hardy inequality (see [9]) that $\left|u_{m}\right|^{2^{*}(s)-2} u_{m}$ is bounded in $L^{2^{*}(s) /\left(2^{*}(s)-1\right)}\left(\mathbb{R}^{N},|x|^{-s}\right)$, thus we have that

$$
\left|u_{m}\right|^{2^{*}(s)-2} u_{m} \rightarrow|u|^{2^{*}(s)-2} u \text { weakly in } L^{2^{*}(s) /\left(2^{*}(s)-1\right)}\left(\mathbb{R}^{N},|x|^{-s}\right) .
$$

Since $J^{\prime}\left(u_{m}\right) \rightarrow 0$, from $(2.36)$ and (2.37), we obtain

$$
\left\langle J^{\prime}(u), u\right\rangle=\|u\|^{2}-\int \frac{|u|^{2^{*}(s)}}{|x|^{s}} d x-\int a(x)|u|^{r} d x-\lambda \int|u|^{2} d x=\lim _{m \rightarrow \infty}\left\langle J^{\prime}\left(u_{m}\right), u\right\rangle=0 .
$$

Set $v_{m} \equiv u_{m}-u$, by Brezis-Lieb lemma [2], we have

$$
\begin{gathered}
\left\|u_{m}\right\|^{2}=\left\|v_{m}\right\|^{2}+\|u\|^{2}+o(1) \\
\int \frac{\left|u_{m}\right|^{2^{*}(s)}}{|x|^{s}} d x=\int \frac{|u|^{2^{*}(s)}}{|x|^{s}} d x+\int \frac{\left|v_{m}\right|^{2^{*}(s)}}{|x|^{s}} d x+o(1) .
\end{gathered}
$$


It follows directly from (2.31)-(2.40) that

$$
\begin{aligned}
o(1)\left\|u_{m}\right\| & =\left\langle J^{\prime}\left(u_{m}\right), u_{m}\right\rangle=\left\|u_{m}\right\|^{2}-\int \frac{\left|u_{m}\right|^{2^{*}(s)}}{|x|^{s}} d x-\int a(x)\left|u_{m}\right|^{r} d x-\lambda \int\left|u_{m}\right|^{2} d x \\
& =\left\langle J^{\prime}(u), u\right\rangle+\left\|v_{m}\right\|^{2}-\int \frac{\left|v_{m}\right|^{2^{*}(s)}}{|x|^{s}} d x+o(1)=\left\|v_{m}\right\|^{2}-\int \frac{\left|v_{m}\right|^{2^{*}(s)}}{|x|^{s}} d x+o(1) \\
J(u) & =J\left(u_{m}\right)-\frac{1}{2}\left\|v_{m}\right\|^{2}+\frac{1}{2^{*}(s)} \int \frac{\left|v_{m}\right|^{2^{*}(s)}}{|x|^{s}} d x+o(1) \\
& =c-\frac{1}{2}\left\|v_{m}\right\|^{2}+\frac{1}{2^{*}(s)} \int \frac{\left|v_{m}\right|^{2^{*}(s)}}{|x|^{s}} d x+o(1) .
\end{aligned}
$$

Since $\left\{\left\|v_{m}\right\|\right\}$ is bounded, without loss of generality, we may assume that

$$
\lim _{m \rightarrow \infty}\left\|v_{m}\right\|^{2}=k
$$

Then we get that

$$
\lim _{m \rightarrow \infty} \int \frac{\left|v_{m}\right|^{2^{*}(s)}}{|x|^{s}} d x=k
$$

By the Sobolev-Hardy inequality,

$$
\int \frac{\left|v_{m}\right|^{2^{*}(s)}}{|x|^{s}} d x \leq A_{s}^{-2^{*}(s) / 2}\left\|v_{m}\right\|^{2^{*}(s)}
$$

for all $m \in N$. Then by taking $m \rightarrow+\infty$, we obtain

$$
k \leq A_{s}^{-2^{*}(s) / 2} k^{2^{*}(s) / 2} .
$$

If $k>0$, we have that $k \geq A_{s}^{2^{*}(s) /\left(2^{*}(s)-2\right)}$. By $(2.41)$ we deduce that

$$
J(u)=c-\left(\frac{1}{2}-\frac{1}{2 *(s)}\right) k \leq c-\frac{2^{*}(s)-2}{22^{*}(s)} A_{s}^{2^{*}(s) /\left(2^{*}(s)-2\right)}=c-\frac{2-s}{2(N-s)} A_{s}^{(N-s)(2-s)}<0,
$$

but from (2.38), we get

$$
J(u)=J(u)-\frac{1}{2}\left\langle J^{\prime}(u), u\right\rangle=\left(\frac{1}{2}-\frac{1}{2 *(s)}\right) \int \frac{|u|^{2^{*}(s)}}{|x|^{s}} d x+\left(\frac{1}{2}-\frac{1}{r}\right) \int a(x)|u|^{r} d x \geq 0,
$$

this contradiction implies $k=0$. By the definition of $v_{m}$, we conclude that $J(u)$ satisfies $(\mathrm{PS})_{c}$ condition. We have completed the proof of Lemma 2.7. 
Proof of Theorem 1.1. By the Sobolev-Hardy inequality and Lemma 2.4, for any $u \in H_{r} \backslash\{0\}$, we have

$$
\begin{aligned}
J(u) & =\frac{1}{2}\|u\|^{2}-\frac{1}{2^{*}(s)} \int \frac{|u|^{2^{*}(s)}}{|x|^{s}} d x-\frac{1}{r} \int a(x)|u|^{r} d x-\frac{\lambda}{2} \int|u|^{2} d x \\
& \geq\left(\frac{1}{2}-\frac{\lambda}{2 \lambda_{1}(\mu)}\right)\|u\|^{2}-\frac{C}{2^{*}(s)}\|u\|^{2^{*}(s)}-\frac{C}{r}\|u\|^{r} \\
& \geq\|u\|^{2}\left(\frac{\lambda_{1}(\mu)-\lambda}{2 \lambda_{1}(\mu)}-C\left(\|u\|^{2^{*}(s)-2}+\|u\|^{r-2}\right)\right) .
\end{aligned}
$$

Clearly, for $\rho>0$ small enough, there exists $\beta>0$ such that $J(u) \geq \beta$ for all $u \in \partial B_{\rho}=\{u \in$ $\left.H_{r},\|u\|=\rho\right\}$. For $u_{0} \in H_{r} \backslash\{0\}, t \geq 0$, we have

$$
J\left(t u_{0}\right)=\frac{t^{2}}{2}|| u_{0} \|^{2}-\frac{t^{2^{*}(s)}}{2 *(s)} \int \frac{\left|u_{0}\right|^{2^{*}(s)}}{|x|^{s}} d x-\frac{t^{r}}{r} \int a(x)\left|u_{0}\right|^{r} d x-\frac{\lambda t^{2}}{2} \int\left|u_{0}\right|^{2} d x .
$$

Obviously, $\lim _{t \rightarrow+\infty} J\left(t u_{0}\right)=-\infty$, so we may choose $t_{0}$ large enough, such that $\left\|t_{0} u_{0}\right\|>$ $\left\|u_{0}\right\|=\rho$ for some $u_{0} \in \partial B_{\rho}$, and $J\left(t_{0} u_{0}\right)<0$. By Lemmas 2.6 and 2.7 and the mountain pass theorem given in [1] (or [3]), we get a sequence $\left\{u_{m}\right\} \subset H_{r}, u_{m} \rightarrow u$ strongly for some $u \in H_{r}$, and $J(u)=c, J^{\prime}(u)=0$. Thus $u$ is a nontrivial solution of problem (1.1). we have finished the proof of Theorem 1.1.

Remark 2.8. If $\lambda=0$, using similar ways, we can prove that problem (1.1) has at least a nontrivial solution in $H$ when $r, \mu$ satisfy the condition (i) of Theorem 1.1.

\section{Acknowledgment}

This research was supported by National Natural Science Foundation of China under Grant 10271077, Science and Technology Committee of Shanghai under Grant 03JC14013, and National Natural Science Foundation of China (Mathematics Tianyuan Youth Foundation) under Grant A0324610.

\section{References}

[1] A. Ambrosetti and P. H. Rabinowitz, Dual variational methods in critical point theory and applications, J. Functional Analysis 14 (1973), no. 4, 349-381.

[2] H. Brézis and E. H. Lieb, A relation between pointwise convergence of functions and convergence of functionals, Proc. Amer. Math. Soc. 88 (1983), no. 3, 486-490.

[3] H. Brézis and L. Nirenberg, Positive solutions of nonlinear elliptic equations involving critical Sobolev exponents, Comm. Pure Appl. Math. 36 (1983), no. 4, 437-477.

[4] D. Cao and P. Han, Solutions for semilinear elliptic equations with critical exponents and Hardy potential, J. Differential Equations 205 (2004), no. 2, 521-537.

[5] D. Cao and S. Peng, A note on the sign-changing solutions to elliptic problems with critical Sobolev and Hardy terms, J. Differential Equations 193 (2003), no. 2, 424-434.

[6] J. Chen, Existence of solutions for a nonlinear PDE with an inverse square potential, J. Differential Equations 195 (2003), no. 2, 497-519.

[7] A. Ferrero and F. Gazzola, Existence of solutions for singular critical growth semilinear elliptic equations, J. Differential Equations 177 (2001), no. 2, 494-522. 
[8] J. P. García Azorero and I. Peral Alonso, Hardy inequalities and some critical elliptic and parabolic problems, J. Differential Equations 144 (1998), no. 2, 441-476.

[9] N. Ghoussoub and C. Yuan, Multiple solutions for quasi-linear PDEs involving the critical Sobolev and Hardy exponents, Trans. Amer. Math. Soc. 352 (2000), no. 12, 5703-5743.

[10] E. Jannelli, The role played by space dimension in elliptic critical problems, J. Differential Equations 156 (1999), no. 2, 407-426.

[11] D. Kang and Y. Deng, Existence of solution for a singular critical elliptic equation, J. Math. Anal. Appl. 284 (2003), no. 2, 724-732.

[12] D. Kang and S. Peng, Positive solutions for singular critical elliptic problems, Appl. Math. Lett. 17 (2004), no. 4, 411-416.

[13] W. A. Strauss, Existence of solitary waves in higher dimensions, Comm. Math. Phys. 55 (1977), no. $2,149-162$.

Juan Li: Department of Mathematics, Shanghai Jiaotong University, Shanghai 200240, China E-mail addresses: juanjuan-li@sjtu.edu.cn; juanjuan-li@163.com 


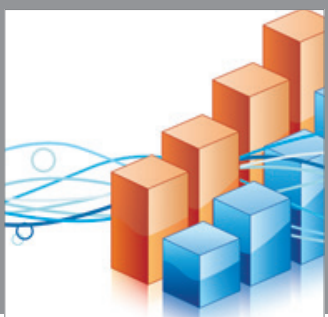

Advances in

Operations Research

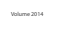

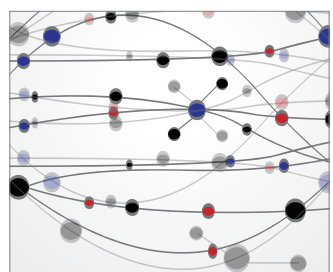

\section{The Scientific} World Journal
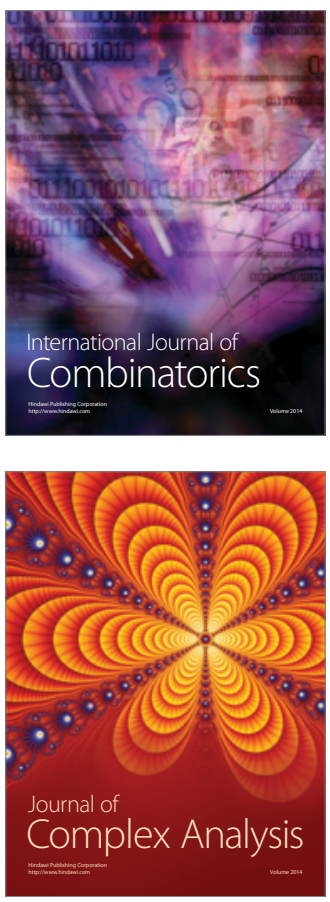

International Journal of

Mathematics and

Mathematical

Sciences
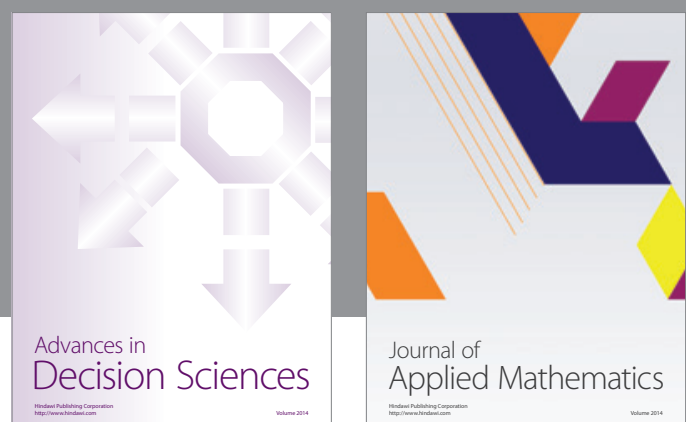

Journal of

Applied Mathematics
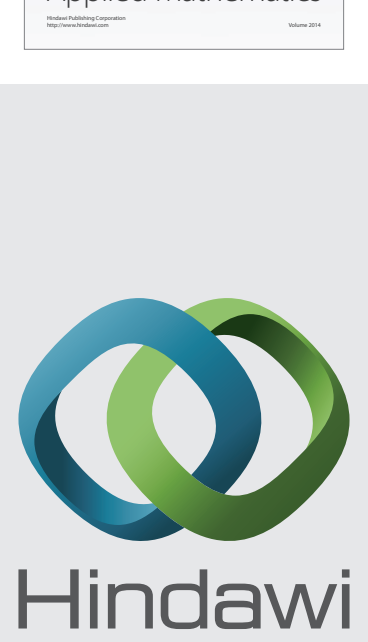

Submit your manuscripts at http://www.hindawi.com
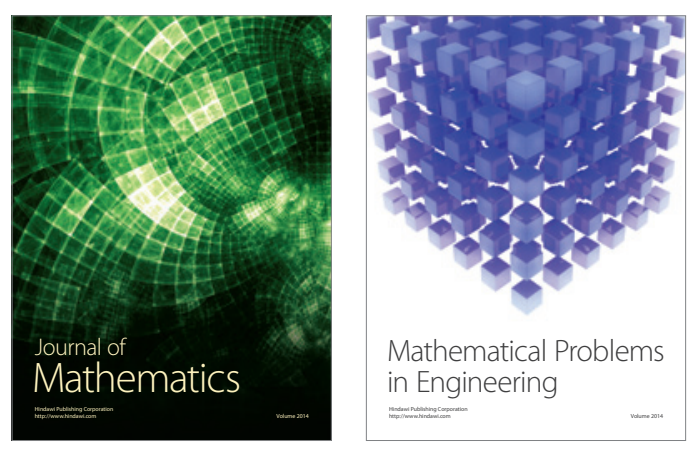

Mathematical Problems in Engineering
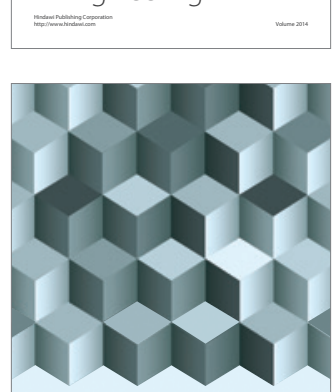

Journal of

Function Spaces
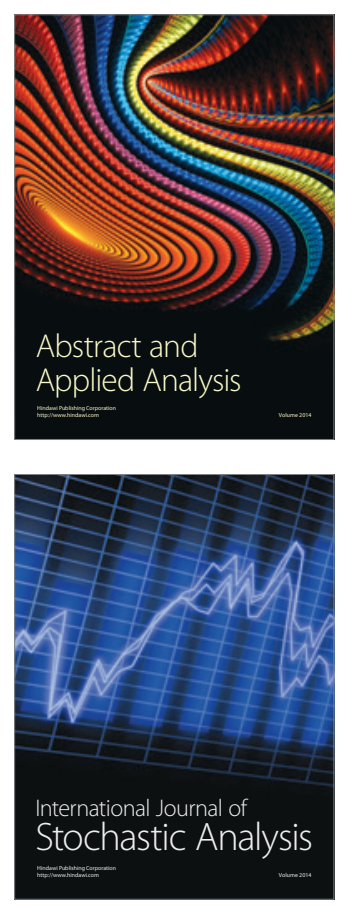

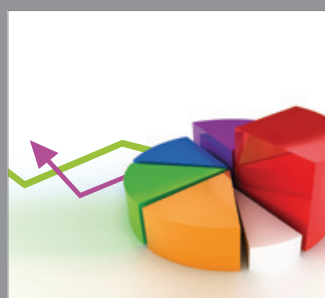

ournal of

Probability and Statistics

Promensencen
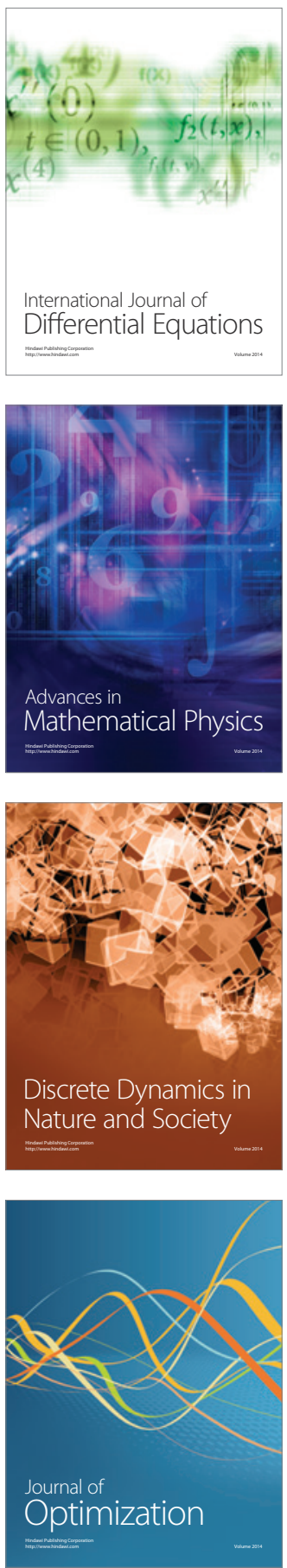